\title{
Scattering of a Diagonally Incident Focused Gaussian Beam by an Infinitely Long Homogeneous Circular Cylinder
}

James A. Lock

Cleveland State University, j.lock@csuohio.edu

Follow this and additional works at: https://engagedscholarship.csuohio.edu/sciphysics_facpub

Part of the Physics Commons

How does access to this work benefit you? Let us know!

\section{Publisher's Statement}

This paper was published in Journal of the Optical Society of America A: Optics Image Science and Vision and is made available as an electronic reprint with the permission of OSA. The paper can be found at the following URL on the OSA website: http://www.opticsinfobase.org/josaa/ abstract.cfm?URI=josaa-14-3-640. Systematic or multiple reproduction or distribution to multiple locations via electronic or other means is prohibited and is subject to penalties under law.

\section{Original Citation}

Lock, James A. "Scattering of a Diagonally Incident Focused Gaussian Beam by an Infinitely Long Homogeneous Circular Cylinder." Journal of the Optical Society of America A: Optics Image Science and Vision 14 (1997): 640-652.

\section{Repository Citation}

Lock, James A., "Scattering of a Diagonally Incident Focused Gaussian Beam by an Infinitely Long Homogeneous Circular Cylinder" (1997). Physics Faculty Publications. 69.

https://engagedscholarship.csuohio.edu/sciphysics_facpub/69

This Article is brought to you for free and open access by the Physics Department at EngagedScholarship@CSU. It has been accepted for inclusion in Physics Faculty Publications by an authorized administrator of EngagedScholarship@CSU. For more information, please contact library.es@csuohio.edu. 


\title{
Scattering of a diagonally incident focused Gaussian beam by an infinitely long homogeneous circular cylinder
}

\author{
James A. Lock \\ Department of Physics, Cleveland State University, Cleveland, Ohio 44115
}

Received April 11, 1996; revised manuscript received September 23, 1996; accepted October 7, 1996

I expand the radiation potential of an arbitrary monochromatic electromagnetic wave in the cylindrical coordinate eigenfunctions of the scalar Helmholtz equation. Since the resulting beam shape coefficients are found to be an inverse Fourier transform of the $z$ component of the beam fields, the incident Gaussian beam is parameterized by a Fourier angular spectrum of plane waves. The beam's partial-wave coefficients are then obtained, as well as the scattered fields produced by the interaction of the beam with an infinitely long homogeneous circular cylinder. The fields are evaluated analytically in the far zone by the method of stationary phase, and the physical interpretation of the results are discussed extensively. (c) 1997 Optical Society of America [S0740-3232(97)01203-9]

\section{INTRODUCTION}

In the branch of light-scattering theory known as LorenzMie theory the electromagnetic boundary-value problem of the interaction of a linearly polarized monochromatic plane wave with a spherical particle is solved exactly. The resulting expressions for the scattered electric and magnetic fields contain a slowly convergent infinite series whose terms are complicated combinations of spherical Bessel, Neumann, and Hankel functions, and angular functions that are related to associated Legendre polynomials. $^{1-3}$ The mathematical complexity of this exact solution obscures its physical interpretation. Over the years many mathematical techniques such as the Debye series, ${ }^{4,5}$ the Watson transform, ${ }^{6,7}$ and stationaryphase arguments ${ }^{8}$ have been employed in order to make explicit the physical interpretation of the scattering that is implicit in the Lorenz-Mie theory formulas.

The situation becomes more complicated for scattering of a diagonally incident plane wave by an infinitely long homogeneous circular cylinder. Mathematically, the scattering amplitudes contain many more Bessel, Neumann, and Hankel functions than for sphere scattering. ${ }^{9-11}$ Physically, the cylinder geometry permits additional phenomena to occur, such as cross-polarized scattering, that were impossible for sphere scattering. The complexity of the scattering amplitudes has hindered efforts to obtain a physical intuition of diagonal-incidence cylinder scattering that is as refined as is our intuition for plane-wave-sphere scattering.

A number of years ago, the theory of scattering a normally incident two-dimensional Gaussian laser sheet by an infinitely long circular cylinder by using a Fourier series expansion or an angular spectrum of plane waves model for the incident beam was developed by Alexopoulos and Park, ${ }^{12}$ Kojima and Yanagiuchi, ${ }^{13}$ and Kozaki. ${ }^{14,15}$ The angular spectrum of plane waves model was subsequently extended to off-center incidence, ${ }^{16,17}$ incidence on a layered cylinder, ${ }^{18,19}$ and incidence on an array of cylinders. ${ }^{20}$ The incident Gaussian laser sheet scattering problem is similar to the incident focused Gaussianbeam scattering problem, except that the former is a twodimensional problem while the latter is intrinsically a three-dimensional problem. Recently, a solution to the focused Gaussian-beam-cylinder problem has been published $^{21-27}$ that employs the Davis-Barton model ${ }^{28,29}$ for the incident beam and uses the theory of distributions to calculate the incident-beam coefficients. The DavisBarton beam model has been highly successful in describing focused Gaussian-beam-sphere scattering ${ }^{30-36}$ and leads, through the theory of distributions, to a consistent solution to the focused Gaussian-beam-cylinder problem. In this paper it is demonstrated that modeling the incident beam by an angular spectrum of plane waves ${ }^{14,37-39}$ provides what I feel to be a more intuitive and easily interpretable approach to the Gaussian-beam-cylinder scattering problem.

The body of this paper proceeds as follows. Section 2 presents the separation-of-variables solution to the scattering problem. In Subsection 2.A the $\epsilon$ and $\mu$ polarization states of the beam are defined. In Subsection 2.B the method of solution is outlined, and general results are presented. In Section 3 the formulas for diagonal planewave-cylinder scattering are briefly presented, since the solution is important for understanding the solution to the diagonally incident Gaussian-beam-cylinder problem. In Section 4 we examine the beam shape coefficients for three different models of a focused Gaussian beam: the Davis-Barton model in Subsection 4.A, the angular spectrum of plane waves model in Subsection 4.B, and the coefficient specification model using the localized approximation in Subsection 4.C. In Section 5 we use the localized beam shape coefficients to obtain an analytical approximation to the far-zone scattered fields. Finally, Section 6 clarifies the relation between the scat- 
tered fields for diagonal plane-wave incidence and those for diagonal Gaussian-beam incidence.

\section{GENERAL SOLUTION OF THE ELECTROMAGNETIC-BEAM-CYLINDER- SCATTERING PROBLEM}

\section{A. Definition of the $\epsilon$ and $\mu$ Polarization States}

Consider an infinitely long homogeneous circular cylinder of radius $a$ and refractive index $n$, whose symmetry axis coincides with the $z$ axis of a rectangular coordinate system. The cylindrical coordinates with respect to these axes are $(r, \theta, z)$, as shown in Fig. 1. A monochromatic electromagnetic beam of wave number $k$ and angular frequency $\omega$ is incident on the cylinder. The time dependence of the beam is $\exp (-i \omega t)$ and will hereafter be omitted. The dominant propagation direction of the beam lies in the $x-z$ plane and makes an angle $\xi$ with the $x$ axis, i.e.,

$$
\mathbf{k}_{\text {inc }}=k\left[(\cos \xi) \hat{u}_{x}-(\sin \xi) \hat{u}_{z}\right]
$$

The fields of the incident beam, as well as those of the scattered and interior fields produced by the interaction of the incident beam with the cylinder, have two orthogonal polarization states. We use the notation $\epsilon$ and $\mu$ in this paper to denote the polarization states (rather than TE and TM) in order to avoid the following notational conflict. In sphere scattering $\mathrm{TE}$ is associated with the partial-wave scattering amplitude $b_{l}$, and TM is associated with $a_{l}$. In cylinder scattering, if we were to use TE and TM, we would end up associating TE with $a_{l}$ and TM with $b_{l}$

In what we call the $\epsilon$ polarization (case II in Ref. 40 , case II or TE in Ref. 41, and case II in Ref. 11) the electric field is confined to the horizontal $(x-y)$ plane in Fig. 1, and the fields may be written as

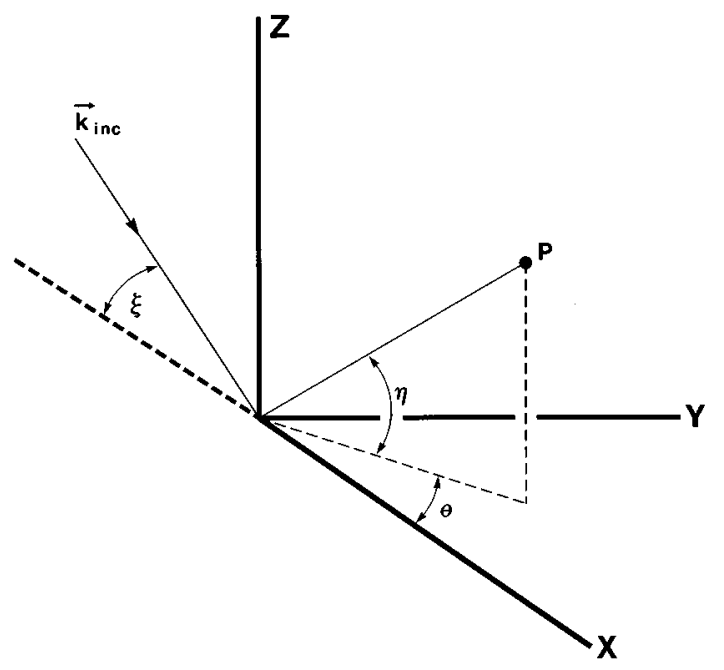

Fig. 1. Geometry of the incident beam and the scattered wave. The dominant incident propagation vector lies in the $x-z$ plane and makes an angle $\xi$ with the $x$ axis. The cylindrical coordinates of the point $P$ in the far zone of the scattered radiation are $(r, \theta, z)$

$$
\begin{aligned}
\mathbf{E}(r, \theta, z)= & -\hat{u}_{z} \times \nabla \psi^{\epsilon}(r, \theta, z) \\
= & \frac{1}{r} \frac{\partial \psi^{\epsilon}}{\partial \theta} \hat{u}_{r}-\frac{\partial \psi^{\epsilon}}{\partial r} \hat{u}_{\theta}, \\
\mathbf{B}(r, \theta, z)= & \frac{i}{\omega} \nabla \times\left[\hat{u}_{z} \times \nabla \psi^{\epsilon}(r, \theta, z)\right] \\
= & \frac{-i}{\omega} \frac{\partial^{2} \psi^{\epsilon}}{\partial r \partial z} \hat{u}_{r}-\frac{i}{\omega r} \frac{\partial^{2} \psi^{\epsilon}}{\partial \theta \partial z} \hat{u}_{\theta} \\
& -\frac{i}{\omega}\left(N^{2} k^{2} \psi^{\epsilon}+\frac{\partial^{2} \psi^{\epsilon}}{\partial z^{2}}\right) \hat{u}_{z},
\end{aligned}
$$

where $N$ is the refractive index of the medium (i.e., $N$ $=n$ inside the cylinder and $N=1$ outside) and $\psi^{\epsilon}$ is the $\epsilon$ polarization radiation potential that satisfies the scalar Helmholtz equation. The radiation potential of the incident beam may be written as $^{21}$

$$
\begin{aligned}
\psi_{\mathrm{inc}}^{\epsilon}(r, \theta, z)= & \int_{-\infty}^{\infty} \mathrm{d} h \sum_{l=-\infty}^{\infty} \frac{i^{l+1}}{k \cos \xi} E_{0} A_{l}(h) \\
& \times J_{l}\left[k r\left(1-h^{2}\right)^{1 / 2}\right] \\
& \times \exp (i k h z) \exp (i l \theta),
\end{aligned}
$$

where $h$ and $l$ are the continuous and discrete cylindrical coordinate separation variables, respectively. The variable $l$ is known as the partial wave number. For an incident plane wave the variable $h$ takes on only a single value. For a more general incident beam $h$ has a spectrum of values. Also in Eq. (3), $J_{l}$ are Bessel functions, $E_{0}$ is a measure of the peak electric-field strength of the beam, and $A_{l}(h)$ are the partial-wave beam shape coefficients. The expressions for $\psi^{\epsilon}$ for both the scattered and interior fields are given in Subsection 2.B. If the fields of the incident beam are known exactly, the beam shape coefficients are obtained by substituting Eq. (3) into Eqs. (2) to produce $^{21}$

$$
\begin{aligned}
A_{l}(h)= & \frac{(-i)^{l} k \cos \xi}{(2 \pi)^{2}\left(1-h^{2}\right) J_{l}\left[k r\left(1-h^{2}\right)^{1 / 2}\right]} \\
& \times \int_{-\infty}^{\infty} \mathrm{d} z \int_{0}^{2 \pi} \mathrm{d} \theta[\exp (-i k h z) \\
& \times \exp (-i l \theta)] c B_{z, \text { inc }}(r, \theta, z) / E_{0},
\end{aligned}
$$

where $B_{z \text {,inc }}$ is the $z$ component of the incident magnetic field and $c$ is the speed of light. If the beam fields are an exact solution of Maxwell's equations, the $r$ dependence in Eq. (4) cancels ${ }^{33}$ and the beam shape coefficients are functions of $h$ and $l$ alone.

In what we call the $\mu$ polarization (case I in Ref. 40, case I or TM in Ref. 41, and case I in Ref. 11) the magnetic field is confined to the horizontal $(x-y)$ plane in Fig. 1, and the fields may be written as 


$$
\begin{aligned}
\mathbf{E}(r, \theta, z)= & \frac{i c}{N \omega} \nabla \times\left[\hat{u}_{z} \times \nabla \psi^{\mu}(r, \theta, z)\right] \\
= & \frac{-i c}{N \omega} \frac{\partial^{2} \psi^{\mu}}{\partial r \partial z} \hat{u}_{r}-\frac{i c}{N \omega r} \frac{\partial^{2} \psi^{\mu}}{\partial \theta \partial z} \hat{u}_{\theta} \\
& -\frac{i c}{N \omega}\left(N^{2} k^{2} \psi^{\mu}+\frac{\partial^{2} \psi^{\mu}}{\partial z^{2}}\right) \hat{u}_{z}, \\
\mathbf{B}(r, \theta, z, t)= & \frac{N}{c} \hat{u}_{z} \times \nabla \psi^{\mu}(r, \theta, z) \\
= & -\frac{N}{c r} \frac{\partial \psi^{\mu}}{\partial \theta} \hat{u}_{r}+\frac{N}{c} \frac{\partial \psi^{\mu}}{\partial r} \hat{u}_{\theta},
\end{aligned}
$$

where $\psi^{\mu}$ is the $\mu$ polarization radiation potential. The radiation potential of the incident beam may be written as

$$
\begin{aligned}
\psi_{\text {inc }}^{\mu}(r, \theta, z)= & \int_{-\infty}^{\infty} \mathrm{d} h \sum_{l=-\infty}^{\infty} \frac{i^{l+1}}{k \cos \xi} E_{0} B_{l}(h) \\
& \times J_{l}\left(k r\left(1-h^{2}\right)^{1 / 2}\right) \\
& \times \exp (i k h z) \exp (i l \theta)
\end{aligned}
$$

where $B_{l}(h)$ are the beam shape coefficients. The expressions for $\psi^{\mu}$ for both the scattered and interior fields are also given in Subsection 2.B. Again, if the incidentbeam fields are known exactly, the beam shape coefficients are obtained by substituting Eq. (6) into Eqs. (5) to produce

$$
\begin{aligned}
B_{l}(h)= & \frac{(-i)^{l} k \cos \xi}{(2 \pi)^{2}\left(1-h^{2}\right) J_{l}\left(k r\left(1-h^{2}\right)^{1 / 2}\right)} \\
& \times \int_{-\infty}^{\infty} \mathrm{d} z \int_{0}^{2 \pi} \mathrm{d} \theta[\exp (-i k h z) \\
& \times \exp (-i l \theta)] E_{z, \text { inc }}(r, \theta, z) / E_{0},
\end{aligned}
$$

where $E_{z \text {,inc }}$ is the $z$ component of the incident electric field.

\section{B. Separation-of-Variables Solution and the Far-Zone Scattered Fields}

In this subsection the separation-of-variables solution of the diagonally incident beam-cylinder scattering problem is briefly outlined, and all the general formulas describing the far-zone scattered fields and intensity are collected together. The $\epsilon$-polarized and $\mu$-polarized scattered radiation potentials are taken to be

$$
\begin{aligned}
\psi_{\text {scatt }}^{\epsilon}(r, \theta, z)= & -\int_{-\infty}^{\infty} \mathrm{d} h \sum_{l=-\infty}^{\infty} \frac{i^{l+1}}{k \cos \xi} \\
& \times E_{0} \alpha_{l}(h) H_{l}^{(1)}\left(k r\left(1-h^{2}\right)^{1 / 2}\right) \\
& \times \exp (i k h z) \exp (i l \theta)
\end{aligned}
$$

$$
\begin{aligned}
\psi_{\text {scatt }}^{\mu}(r, \theta, z)= & -\int_{-\infty}^{\infty} \mathrm{d} h \sum_{l=-\infty}^{\infty} \frac{i^{l+1}}{k \cos \xi} \\
& \times E_{0} \beta_{l}(h) H_{l}^{(1)}\left(k r\left(1-h^{2}\right)^{1 / 2}\right) \\
& \times \exp (i k h z) \exp (i l \theta),
\end{aligned}
$$

where $H_{l}^{(1)}$ are Hankel functions of the first kind and $\alpha_{l}(h)$ and $\beta_{l}(h)$ are the partial-wave scattering amplitudes. The interior radiation potentials are

$$
\begin{aligned}
\psi_{\text {interior }}^{\epsilon}(r, \theta, z)= & \int_{-\infty}^{\infty} \mathrm{d} h^{\prime} \sum_{l=-\infty}^{\infty} \frac{i^{l+1}}{k \cos \xi} \\
& \times E_{0} n \gamma_{l}\left(h^{\prime}\right) J_{l}\left(n k r\left(1-h^{\prime 2}\right)^{1 / 2}\right) \\
& \times \exp \left(i n k h^{\prime} z\right) \exp (i l \theta), \\
\psi_{\text {interior }}^{\mu}(r, \theta, z)= & \int_{-\infty}^{\infty} \mathrm{d} h^{\prime} \sum_{l=-\infty}^{\infty} \frac{i^{l+1}}{k \cos \xi} \\
& \times E_{0} n \delta_{l}\left(h^{\prime}\right) J_{l}\left(n k r\left(1-h^{\prime 2}\right)^{1 / 2}\right) \\
& \times \exp \left(i n k h^{\prime} z\right) \exp (i l \theta),
\end{aligned}
$$

where $\gamma_{l}\left(h^{\prime}\right)$ and $\delta_{l}\left(h^{\prime}\right)$ are the partial-wave interior amplitudes. If the equations describing the continuity of the tangential components of the total electric and magnetic fields at the surface of the cylinder are to be independent of $z$, we require that

$$
h^{\prime}=h / n \text {. }
$$

The solution of the four field continuity conditions in the four unknowns $\alpha_{l}(h), \beta_{l}(h), \gamma_{l}\left(h^{\prime}\right)$, and $\delta_{l}\left(h^{\prime}\right)$ then gives $^{21}$

$$
\begin{aligned}
& \alpha_{l}(h)=a_{l}(h) A_{l}(h)+q_{l}(h) B_{l}(h), \\
& \beta_{l}(h)=-q_{l}(h) A_{l}(h)+b_{l}(h) B_{l}(h)
\end{aligned}
$$

for the partial-wave scattering amplitudes and

$$
\begin{aligned}
& \gamma_{l}\left(h^{\prime}\right)=c_{l}(h) A_{l}(h)+p_{l}(h) B_{l}(h), \\
& \delta_{l}\left(h^{\prime}\right)=-n p_{l}(h) A_{l}(h)+d_{l}(h) B_{l}(h)
\end{aligned}
$$

for the partial-wave interior amplitudes, where

$$
\begin{aligned}
x \equiv k a\left(1-h^{2}\right)^{1 / 2}, \quad y \equiv n k a\left(1-h^{2} / n^{2}\right)^{1 / 2}, \\
a_{l}(h)=\frac{U_{2} W_{1}-n U_{3} W_{3}}{W_{1} W_{2}-n W_{3}^{2}}, \\
b_{l}(h)=\frac{U_{1} W_{2}-n U_{3} W_{3}}{W_{1} W_{2}-n W_{3}^{2}}, \\
q_{l}(h)=\frac{2 n l h\left(y^{2}-x^{2}\right)}{\pi x^{2} y^{2}} \frac{J_{l}^{2}(y)}{W_{1} W_{2}-n W_{3}^{2}}, \\
c_{l}(h)=\frac{-2 i n x}{\pi y^{2}} \frac{W_{1}}{W_{1} W_{2}-n W_{3}^{2}}, \\
d_{l}(h)=\frac{-2 i n x}{\pi y^{2}} \frac{W_{2}}{W_{1} W_{2}-n W_{3}^{2}}, \\
p_{l}(h)=\frac{-2 n x}{\pi y^{2}} \frac{W_{3}}{W_{1} W_{2}-n W_{3}^{2}},
\end{aligned}
$$




$$
\begin{aligned}
& U_{1}=\frac{n^{2} x}{y} J_{l}(x) J_{l}^{\prime}(y)-J_{l}^{\prime}(x) J_{l}(y), \\
& U_{2}=\frac{n x}{y} J_{l}(x) J_{l}^{\prime}(y)-n J_{l}^{\prime}(x) J_{l}(y), \\
& U_{3}=\frac{h l\left(y^{2}-x^{2}\right)}{x y^{2}} J_{l}(x) J_{l}(y), \\
& W_{1}=\frac{n^{2} x}{y} H_{l}^{(1)}(x) J_{l}^{\prime}(y)-H_{l}^{(1)^{\prime}}(x) J_{l}(y), \\
& W_{2}=\frac{n x}{y} H_{l}^{(1)}(x) J_{l}^{\prime}(y)-n H_{l}^{(1)^{\prime}}(x) J_{l}(y), \\
& W_{3}=\frac{h l\left(y^{2}-x^{2}\right)}{x y^{2}} H_{l}^{(1)}(x) J_{l}(y) .
\end{aligned}
$$

In Eqs. (11)-(15) the partial-wave amplitudes $a_{l}(h)$ and $c_{l}(h)$ correspond to polarization-preserving scattering, i.e., $\epsilon$ polarization in goes to $\epsilon$ polarization out, which we denote as $\epsilon \epsilon$. Similarly, the partial-wave amplitudes $b_{l}(h)$ and $d_{l}(h)$ correspond to $\mu \mu$ polarization-preserving scattering. The partial-wave amplitudes $q_{l}(h)$ and $p_{l}(h)$ correspond to $\mu \epsilon$ and $\epsilon \mu$ cross-polarized scattering, which occurs only for $h \neq 0$. The far-zone scattered fields are obtained by substituting Eqs. (8) and (11) into Eqs. (2) and (5) and then taking the $r \rightarrow \infty$ limit:

$$
\begin{aligned}
\lim _{r \rightarrow \infty} \mathbf{E}_{\text {scatt }}(r, \theta, z)= & \frac{E_{0}}{\cos \xi}\left(\frac{2}{\pi k r}\right)^{1 / 2}[\exp (-i \pi / 4)] \\
& \times\left(T_{5} \hat{u}_{r}-T_{2} \hat{u}_{\theta}-T_{3} \hat{u}_{z}\right),
\end{aligned}
$$

$\lim _{r \rightarrow \infty} \mathbf{B}_{\text {scatt }}(r, \theta, z)=\frac{E_{0}}{c \cos \xi}\left(\frac{2}{\pi k r}\right)^{1 / 2}[\exp (-i \pi / 4)]$

$$
\times\left(T_{6} \hat{u}_{r}+T_{1} \hat{u}_{\theta}-T_{4} \hat{u}_{z}\right)
$$

where

$$
\begin{aligned}
T_{1}= & \int_{-\infty}^{\infty} \mathrm{d} h \sum_{l=-\infty}^{\infty}\left(1-h^{2}\right)^{1 / 4} \beta_{l}(h) \\
& \times \exp \left[i k r\left(1-h^{2}\right)^{1 / 2}\right] \\
& \times \exp (i k h z) \exp (i l \theta), \\
T_{2}= & \int_{-\infty}^{\infty} \mathrm{d} h \sum_{l=-\infty}^{\infty}\left(1-h^{2}\right)^{1 / 4} \alpha_{l}(h) \\
& \times \exp \left[i k r\left(1-h^{2}\right)^{1 / 2}\right] \\
& \times \exp (i k h z) \exp (i l \theta), \\
T_{3}= & \int_{-\infty}^{\infty} \mathrm{d} h \sum_{l=-\infty}^{\infty}\left(1-h^{2}\right)^{3 / 4} \beta_{l}(h) \\
& \times \exp \left[i k r\left(1-h^{2}\right)^{1 / 2}\right] \\
& \times \exp (i k h z) \exp (i l \theta),
\end{aligned}
$$

$$
\begin{aligned}
T_{4}= & \int_{-\infty}^{\infty} \mathrm{d} h \sum_{l=-\infty}^{\infty}\left(1-h^{2}\right)^{3 / 4} \alpha_{l}(h) \\
& \times \exp \left[i k r\left(1-h^{2}\right)^{1 / 2}\right] \\
& \times \exp (i k h z) \exp (i l \theta), \\
T_{5}= & \int_{-\infty}^{\infty} \mathrm{d} h \sum_{l=-\infty}^{\infty} h\left(1-h^{2}\right)^{1 / 4} \beta_{l}(h) \\
& \times \exp \left[i k r\left(1-h^{2}\right)^{1 / 2}\right] \\
& \times \exp (i k h z) \exp (i l \theta), \\
T_{6}= & \int_{-\infty}^{\infty} \mathrm{d} h \sum_{l=-\infty}^{\infty} h\left(1-h^{2}\right)^{1 / 4} \alpha_{l}(h) \\
& \times \exp \left[i k r\left(1-h^{2}\right)^{1 / 2}\right] \\
& \times \exp (i k h z) \exp (i l \theta) .
\end{aligned}
$$

The scattered intensity vector is then

$$
\begin{aligned}
\lim _{r \rightarrow \infty} \mathbf{I}_{\text {scatt }}(r, \theta, z) \\
=\operatorname{Re}\left(\frac{\mathbf{E}_{\text {scatt }}^{*} \times \mathbf{B}_{\text {scatt }}}{2 \mu_{0} c}\right) \\
=\frac{2}{\pi k r \cos ^{2} \xi}\left(\frac{E_{0}^{2}}{2 \mu_{0} c}\right) \operatorname{Re}\left[\left(T_{2}^{*} T_{4}+T_{3}^{*} T_{1}\right) \hat{u}_{r}\right. \\
\left.\quad+\left(T_{5}^{*} T_{4}-T_{3}^{*} T_{6}\right) \hat{u}_{\theta}+\left(T_{5}^{*} T_{1}+T_{2}^{*} T_{6}\right) \hat{u}_{z}\right],
\end{aligned}
$$

where $\mu_{0}$ is the permeability of free space.

\section{SCATTERING OF A DIAGONALLY INCIDENT PLANE WAVE BY A CIRCULAR CYLINDER}

This section briefly summarizes the application of the formalism of Subsection 2.B to a diagonally incident plane wave because we will find in Section 4 that the results for scattering of a focused Gaussian beam bear a significant relationship to the results for scattering of a diagonally incident plane wave. The fields of an $\epsilon$-polarized diagonally incident plane wave are

$$
\begin{aligned}
\mathbf{E}_{\mathrm{inc}}(r, \theta, z)= & \mathbf{E}_{0}\left[\exp \left(i \mathbf{k}_{\mathrm{inc}} \cdot \mathbf{r}\right)\right] \hat{u}_{y}, \\
\mathbf{B}_{\mathrm{inc}}(r, \theta, z)= & \frac{E_{0}}{c}\left[\exp \left(i \mathbf{k}_{\mathrm{inc}} \cdot \mathbf{r}\right)\right] \\
& \times\left[(\sin \xi) \hat{u}_{x}+(\cos \xi) \hat{u}_{z}\right],
\end{aligned}
$$

where $\mathbf{k}_{\text {inc }}$ is given in Eq. (1) and

$$
\mathbf{r}=r(\cos \theta) \hat{u}_{x}+r(\sin \theta) \hat{u}_{y}+z \hat{u}_{z} .
$$

Substitution of Eqs. (20) into Eq. (4) gives

$$
A_{l}(h)=\delta(h+\sin \xi), \quad B_{l}(h)=0,
$$

where $\delta(h+\sin \xi)$ is the Dirac delta function. The fields of a $\mu$-polarized diagonally incident plane wave are 


$$
\begin{aligned}
& \mathbf{E}_{\text {inc }}(r, \theta, z)=E_{0}\left[\exp \left(i \mathbf{k}_{\mathrm{inc}} \cdot \mathbf{r}\right)\right]\left[(\sin \xi) \hat{u}_{x}+(\cos \xi) \hat{u}_{z}\right], \\
& \mathbf{B}_{\text {inc }}(r, \theta, z)=\frac{-E_{0}}{c}\left[\exp \left(i \mathbf{k}_{\mathrm{inc}} \cdot \mathbf{r}\right)\right] \hat{u}_{y} .
\end{aligned}
$$

Substitution of Eqs. (23) into Eq. (7) gives

$$
A_{l}(h)=0, \quad B_{l}(h)=\delta(h+\sin \xi) .
$$

Now that the beam shape coefficients have been determined, the far-zone scattered fields and intensity are easily obtained. Substitution of Eqs. (24) into Eqs. (16)-(19) for a $\mu$-polarized incident plane wave (pw in superscript) gives

$$
\begin{aligned}
& \lim _{r \rightarrow \infty} \mathbf{E}_{\text {scatt }}(r, \theta, z)=\mathbf{E}_{\mu \mu}^{\mathrm{pw}}(r, \theta, z)+\mathbf{E}_{\mu \epsilon}^{\mathrm{pw}}(r, \theta, z), \\
& \lim _{r \rightarrow \infty} \mathbf{B}_{\text {scatt }}(r, \theta, z)=\mathbf{B}_{\mu \mu}^{\mathrm{pw}}(r, \theta, z)+\mathbf{B}_{\mu \epsilon}^{\mathrm{pw}}(r, \theta, z),
\end{aligned}
$$

where the $\mu$-polarized scattered fields are

$$
\begin{aligned}
& \mathbf{E}_{\mu \mu}^{\mathrm{pw}}(r, \theta, z)=\varepsilon_{0}^{\mathrm{pw}}\left[S_{\mu}^{\mathrm{pw}}(\theta)\right]\left[(\sin \xi) \hat{u}_{r}+(\cos \xi) \hat{u}_{z}\right], \\
& \mathbf{B}_{\mu \mu}^{\mathrm{pw}}(r, \theta, z)=\frac{\varepsilon_{0}^{\mathrm{pw}}}{c}\left[S_{\mu}^{\mathrm{pw}}(\theta)\right]\left(-\hat{u}_{\theta}\right)
\end{aligned}
$$

and the $\epsilon$-polarized scattered fields are

$$
\begin{aligned}
& \mathbf{E}_{\mu \epsilon}^{\mathrm{pw}}(r, \theta, z)=\varepsilon_{0}^{\mathrm{pw}}\left[S_{q}^{\mathrm{pw}}(\theta)\right] \hat{u}_{\theta}, \\
& \mathbf{B}_{\mu \epsilon}^{\mathrm{pw}}(r, \theta, z)=\frac{\varepsilon_{0}^{\mathrm{pw}}}{c}\left[S_{q}^{\mathrm{pw}}(\theta)\right]\left[(\sin \xi) \hat{u}_{r}+(\cos \xi) \hat{u}_{z}\right] .
\end{aligned}
$$

In Eqs. (25)-(27) the amplitude and the phase of the scattered fields are

$$
\varepsilon_{0}^{\mathrm{pw}}=-E_{0}\left(\frac{2}{\pi k r \cos \xi}\right)^{1 / 2} \exp (-i \pi / 4) \exp \left(i \mathbf{k}_{\mathrm{scatt}} \cdot \mathbf{r}\right),
$$

the direction of the scattered radiation is

$$
\mathbf{k}_{\text {scatt }}=k\left[(\cos \xi) \hat{u}_{r}-(\sin \xi) \hat{u}_{z}\right],
$$

and the plane-wave angular scattering amplitudes are

$$
\begin{aligned}
S_{\mu}^{\mathrm{pw}}(\theta) & =\sum_{l=-\infty}^{\infty} b_{l}(h=-\sin \xi) \exp (i l \theta) \\
& =b_{0}+2 \sum_{l=1}^{\infty} b_{l} \cos (l \theta), \\
S_{q}^{\mathrm{pw}}(\theta) & =\sum_{l=-\infty}^{\infty} q_{l}(h=-\sin \xi) \exp (i l \theta) \\
& =2 i \sum_{l=1}^{\infty} q_{l} \sin (l \theta) .
\end{aligned}
$$

The far-zone scattered intensity is

$$
\begin{aligned}
\lim _{r \rightarrow \infty} \mathbf{I}_{\text {scatt }}(r, \theta, z)= & \left(\frac{E_{0}{ }^{2}}{2 \mu_{0} c}\right)\left(\frac{2}{\pi k r \cos \xi}\right) \\
& \times\left[\left|S_{\mu}^{\mathrm{pw}}(\theta)\right|^{2}+\left|S_{q}^{\mathrm{pw}}(\theta)\right|^{2}\right] \hat{k}_{\text {scatt }} .
\end{aligned}
$$

Similarly, substitution of Eqs. (22) into Eqs. (16)-(19) for an $\epsilon$-polarized incident plane wave gives

$$
\begin{aligned}
& \lim _{r \rightarrow \infty} \mathbf{E}_{\text {scatt }}(r, \theta, z)=\mathbf{E}_{\epsilon \epsilon}^{\mathrm{pw}}(r, \theta, z)-\mathbf{E}_{\epsilon \mu}^{\mathrm{pw}}(r, \theta, z), \\
& \lim _{r \rightarrow \infty} \mathbf{B}_{\text {scatt }}(r, \theta, z)=\mathbf{B}_{\epsilon \epsilon}^{\mathrm{pw}}(r, \theta, z)-\mathbf{B}_{\epsilon \mu}^{\mathrm{pw}}(r, \theta, z),
\end{aligned}
$$

where the $\epsilon$-polarized scattered fields are

$$
\begin{aligned}
& \mathbf{E}_{\epsilon \epsilon}^{\mathrm{pw}}(r, \theta, z)=\varepsilon_{0}^{\mathrm{pw}}\left[S_{\epsilon}^{\mathrm{pw}}(\theta)\right] \hat{u}_{\theta}, \\
& \mathbf{B}_{\epsilon \epsilon}^{\mathrm{pw}}(r, \theta, z)=\frac{\varepsilon_{0}^{\mathrm{pw}}}{c}\left[S_{\epsilon}^{\mathrm{pw}}(\theta)\right]\left[(\sin \xi) \hat{u}_{r}+(\cos \xi) \hat{u}_{z}\right]
\end{aligned}
$$

and the $\mu$-polarized scattered fields are

$$
\begin{aligned}
& \mathbf{E}_{\epsilon \mu}^{\mathrm{pw}}(r, \theta, z)=\varepsilon_{0}^{\mathrm{pw}}\left[S_{q}^{\mathrm{pw}}(\theta)\right]\left[(\sin \xi) \hat{u}_{r}+(\cos \xi) \hat{u}_{z}\right], \\
& \mathbf{B}_{\epsilon \mu}^{\mathrm{pw}}(r, \theta, z)=\frac{\varepsilon_{0}^{\mathrm{pw}}}{c}\left[S_{q}^{\mathrm{pw}}(\theta)\right]\left(-\hat{u}_{\theta}\right),
\end{aligned}
$$

with

$$
\begin{aligned}
S_{\epsilon}^{\mathrm{pw}}(\theta) & =\sum_{l=-\infty}^{\infty} a_{l}(h=-\sin \xi) \exp (i l \theta) \\
& =a_{0}+2 \sum_{l=1}^{\infty} a_{l} \cos (l \theta) .
\end{aligned}
$$

The far-zone scattered intensity is

$$
\begin{aligned}
\lim _{r \rightarrow \infty} \mathbf{I}_{\text {scatt }}(r, \theta, z)= & \left(\frac{E_{0}{ }^{2}}{2 \mu_{0} c}\right)\left(\frac{2}{\pi k r \cos \xi}\right)\left[\left|S_{\epsilon}^{\mathrm{pw}}(\theta)\right|^{2}\right. \\
& \left.+\left|S_{q}^{\mathrm{pw}}(\theta)\right|^{2}\right] \hat{k}_{\text {scatt }} .
\end{aligned}
$$

Since $q_{l}(h)$ in Eqs. (14) and $S_{q}^{\mathrm{pw}}(\theta)$ in Eqs. (30) are proportional to $h$, cross-polarized scattering occurs only for diagonal incidence $\left(\xi \neq 0^{\circ}\right)$. As was pointed out in Refs. 10, 11, and 42, the scattered wave vector of Eq. (29) lies on the surface of a cone whose symmetry axis coincides with the cylinder's symmetry axis and whose opening half-angle is $\pi / 2-\xi$.

\section{BEAM SHAPE COEFFICIENTS FOR A DIAGONALLY INCIDENT GAUSSIAN BEAM}

The most troublesome aspect of mathematically modeling a focused Gaussian beam is specifying fields that simultaneously produce the required beam shape while also being an exact solution of Maxwell's equations. There are three common ways to accomplish this. Davis ${ }^{28}$ and Barton and Alexander ${ }^{29}$ have outlined a procedure that constructs the fields as an infinite series. The first term of the series is the fields that arise from Fresnel diffracting a Gaussian amplitude profile and a flat phase profile downstream from the beam's focal plane. The $n$ th-order term of the series is the fields that are induced by the spatial variation of the $(n-1)$ st-order fields. This procedure has the advantage of providing analytical expressions for the fields. But it has the disadvantage that truncating the series at any value of $n$ gives expressions that are not an exact solution of Maxwell's equations. 
Also, if the beam is tightly focused, the series is slowly convergent and the amplitude profile in the focal plane is no longer Gaussian.

As an alternative, a focused Gaussian beam may be modeled as a Fourier angular spectrum of plane waves. $^{38,39}$ Such a beam is guaranteed to be an exact solution of Maxwell's equations, since each of the component plane waves in the spectrum is an exact solution, and the amplitude profile in the focal plane is guaranteed to be Gaussian. But this procedure has the disadvantages that the fields are given in integral form, and for a tightly focused beam the integration includes evanescent fields.

As another alternative, a focused Gaussian beam may be modeled by directly specifying ${ }^{34}$ values for the beam shape coefficients in Eqs. (3) and (6) by using the localized approximation. ${ }^{43-45}$ By definition, such a beam is an exact solution of Maxwell's equations. But it has the disadvantages that one does not possess an analytic form for the fields and that, for a tightly focused beam, the amplitude profile in the focal plane is no longer Gaussian. ${ }^{34,35,45}$

\section{A. Davis-Barton Beam Model}

The Davis-Barton fields have been remarkably successful in describing scattering of a focused Gaussian beam by a spherical particle and have led to much physical insight concerning the details of the scattering. It is therefore disappointing that this beam model does not describe Gaussian-beam-cylinder scattering in as simple a way. Mathematically, the difficulties are the following. Consider a first-order Davis-Barton beam propagating along the $x$ axis with the center of its focal waist at the origin. The beam is normally incident on the cylinder and is on axis $^{34}$ with respect to it. This is the simplest Gaussianbeam-cylinder geometry. For a $\mu$-polarized beam, for example, we have ${ }^{28}$

$$
\begin{aligned}
\mathbf{E}_{\mathrm{inc}}(x, y, z)= & E_{0} \frac{\exp (i k x)}{D} \exp \left[-\left(y^{2}+z^{2}\right) / D w_{0}{ }^{2}\right] \\
& \times\left(\frac{-2 i s z}{w_{0} D} \hat{u}_{x}+\hat{u}_{z}\right), \\
\mathbf{B}_{\text {inc }}(x, y, z)= & E_{0} \frac{\exp (i k x)}{c D} \exp \left[-\left(y^{2}+z^{2}\right) / D w_{0}{ }^{2}\right] \\
& \times\left(\frac{2 i s y}{w_{0} D} \hat{u}_{x}-\hat{u}_{y}\right),
\end{aligned}
$$

where $w_{0}$ is the beam's electric-field half-width in the focal plane,

$$
s \equiv \frac{1}{k w_{0}}
$$

is the beam confinement parameter, and

$$
D=1+2 i s^{2} k x \text {. }
$$

Substitution of the $z$ component of $\mathbf{E}_{\text {inc }}$ into Eq. (7) gives

$$
\begin{aligned}
B_{l}(h)= & \frac{(-i)^{l}}{4 s \pi^{3 / 2}\left(1-h^{2}\right) J_{l}\left(k r\left(1-h^{2}\right)^{1 / 2}\right)} \\
& \times \exp \left(-h^{2} / 4 s^{2}\right) \int_{0}^{2 \pi} \frac{\mathrm{d} \theta}{D^{1 / 2}} \\
& \times \exp \left\{i\left[k r\left(1-\frac{h^{2}}{2}\right) \cos \theta-l \theta\right]\right\} \\
& \times \exp \left(\frac{-s^{2} k^{2} r^{2} \sin ^{2} \theta}{D}\right) .
\end{aligned}
$$

The integrand in Eq. (40) may then be expanded in powers of $s$ and integrated term by term in a manner similar to that for Gaussian-beam-sphere scattering. ${ }^{34}$ But here the results are not as pleasing. Upon integration we obtain

$$
\begin{aligned}
B_{l}(h)= & \frac{\exp \left(-h^{2} / 4 s^{2}\right)}{2 s \pi^{1 / 2}\left(1-h^{2}\right)}\left\{\frac{J_{l}\left[k r\left(1-h^{2} / 2\right)\right]}{J_{l}\left[k r\left(1-h^{2}\right)^{1 / 2}\right]}\right. \\
& \times\left[1-\frac{s^{2} l^{2}}{\left(1-h^{2} / 2\right)^{2}}+O\left(s^{4}\right)\right] \\
& \left.+\frac{k r J_{l}^{\prime}\left[k r\left(1-h^{2} / 2\right)\right]}{J_{l}\left[k r\left(1-h^{2}\right)^{1 / 2}\right]}\left[\frac{s^{2} h^{2} / 2}{1-h^{2} / 2}+O\left(s^{4}\right)\right]\right\} .
\end{aligned}
$$

The terms proportional to $k r J_{l}^{\prime}\left(k r\left(1-h^{2} / 2\right)\right) / J_{l}(k r(1$ $\left.-h^{2}\right)^{1 / 2}$ ) were previously called ${ }^{34}$ nonconstant terms and were due to Eqs. (37) not being an exact solution of Maxwell's equations. But for small $h$ the ratio of Bessel functions is nearly unity and the nonconstant terms are small, giving

$$
\begin{aligned}
B_{l}(h) \approx & \frac{1}{2 s \pi^{1 / 2}\left(1-h^{2}\right)}\left[\exp \left(-h^{2} / 4 s^{2}\right)\right]\left(1-\frac{s^{2} l^{2}}{1-h^{2}}\right) \\
\approx & \frac{1}{2 s \pi^{1 / 2}\left(1-h^{2}\right)} \exp \left(-h^{2} / 4 s^{2}\right) \\
& \quad \times \exp \left[-s^{2} l^{2} /\left(1-h^{2}\right)\right] .
\end{aligned}
$$

Based on our experience with Gaussian-beam-sphere scattering, it is tempting to believe that the Davis-Barton third-order and fifth-order fields ${ }^{29}$ would add additional terms to Eq. (41) that would bring the ratio of Bessel functions closer to unity and would cancel the nonconstant terms for low powers of $s^{2}$. But when the higher-order fields are employed, the expected simplifications do not occur in a clear or recognizable way. In Refs. $21-27$ this problem is circumvented by appealing to the mathematical theory of distributions to replace the beam shape functions $A_{l}(h)$ and $B_{l}(h)$ by beam shape distributions that are then evaluated for the Davis-Barton beam model. Such an approach is anticipated by the plane-wave beam shape coefficients of Eqs. (22) and (24). 
The Davis-Barton third-order correction to Eqs. (37) also introduces a $z$ component to $B_{\text {inc }}$ proportional to $s^{2}$. This is unfortunate in that the beam that we originally wanted to be $\mu$ polarized now becomes an $\epsilon$-polarized plus $\mu$-polarized mixture. This considerably complicates the interpretation of the scattered intensity, since not only will we have cross-polarized effects that are due to the diagonal-incidence geometry, but also we will have crosspolarized effects that are due to the incident beam not being a pure polarization state.

As a result of these complications, we no longer consider the Davis-Barton beam model for cylinder scattering in this paper. But relation (42) illustrates an important difference between sphere scattering and cylinder scattering nonetheless. In the solution to the scalar Helmholtz equation in spherical coordinates there are also two separation constants, the partial wave number $l$ and the azimuthal mode number $m$, with $-l \leqslant m \leqslant l$. For on-axis Gaussian-beam-sphere scattering, only $l$ is needed, producing a substantial simplification of the theory; whereas for off-axis scattering both $l$ and $m$ are required. On the other hand, for on-axis normalincidence Gaussian-beam-cylinder scattering both separation constants $l$ and $h$ are required. As a result, we expect that cylinder scattering of an off-axis, a diagonally incident, or an arbitrarily positioned beam produces no further mathematical complication to the general formulas of Subsection 2.B than we would have already experienced for on-axis normal-incidence scattering.

\section{B. Angular Spectrum of Plane-Wave Model}

In this subsection we model a Gaussian beam by superposition of an angular spectrum of plane waves and then calculate its beam shape coefficients. An examination of Eqs. (4) and (7) suggests why this is a reasonable approach. In these equations the $z$ integral is an inverse Fourier transform of the $z$ component of the electric and magnetic fields, with $h$ being the variable in Fourier transform space conjugate to $z$ in coordinate space. This suggests that if $E_{z \text {,inc }}$ and $B_{z \text {,inc }}$ themselves are constructed as a Fourier superposition of plane waves, then $B_{l}(h)$ and $A_{l}(h)$ should be intimately related to the coefficients of the Fourier superposition.

Consider, for example, a $\mu$-polarized normally incident focused Gaussian beam of the form

$$
\mathbf{E}_{\text {inc }}(x, y, z)=\left[E_{x, \text { inc }}(x, y, z)\right] \hat{u}_{x}+\left[E_{z, \text { inc }}(x, y, z)\right] \hat{u}_{z} .
$$

Let the center of the beam focal waist be located at $\left(x_{0}, y_{0}, 0\right)$, and let the beam profile in the focal plane be given by the Gaussian function

$E_{z, \text { inc }}\left(x_{0}, y, z\right)=E_{0} \exp \left[-\left(y-y_{0}\right)^{2} / w_{0}^{2}\right] \exp \left(-z^{2} / w_{0}^{2}\right)$,

with $E_{x, \text { inc }}$ yet to be specified. Equation (44) may be written as the Fourier integral

$$
\begin{aligned}
E_{z, \text { inc }}\left(x_{0}, y, z\right)= & \frac{E_{0} w_{0}{ }^{2}}{4 \pi} \int_{-\infty}^{\infty} k \mathrm{~d} h_{y} \int_{-\infty}^{\infty} k \mathrm{~d} h_{z} \\
& \times \exp \left(-h_{y}{ }^{2} / 4 s^{2}\right) \exp \left(-h_{z}{ }^{2} / 4 s^{2}\right) \\
& \times \exp \left[i h_{y}\left(y-y_{0}\right)\right] \exp \left(i k h_{z} z\right) .
\end{aligned}
$$

Generalizing this to

$$
\begin{aligned}
E_{z, \text { inc }}(x, y, z)= & \frac{E_{0} w_{0}{ }^{2}}{4 \pi} \int_{-\infty}^{\infty} k \mathrm{~d} h_{y} \int_{-\infty}^{\infty} k \mathrm{~d} h_{z} \\
& \times \exp \left(-h_{y}{ }^{2} / 4 s^{2}\right) \exp \left(-h_{z}{ }^{2} / 4 s^{2}\right) \\
& \times \exp \left[i k h_{x}\left(x-x_{0}\right)\right] \\
& \times \exp \left[i k h_{y}\left(y-y_{0}\right)\right] \exp \left(i k h_{z} z\right)
\end{aligned}
$$

produces a beam that is an exact solution of Maxwell's equations, has a Gaussian amplitude profile in the focal plane, is $\mu$ polarized, and is a superposition of plane waves traveling in different directions. The propagation direction of each component plane wave has the direction cosines $h_{x}, h_{y}$, and $h_{z}$, where

$$
h_{x}=\left(1-h_{y}{ }^{2}-h_{z}{ }^{2}\right)^{1 / 2}
$$

When $h_{y}{ }^{2}+h_{z}{ }^{2}>1$, the component plane waves are evanescent. The electric field of the beam in the $x$ direction may be obtained ${ }^{39}$ from Eq. (46) through substitution into the Maxwell equation

$$
\boldsymbol{\nabla} \cdot \mathbf{E}_{\mathrm{inc}}=0 .
$$

In order to evaluate the beam shape coefficients for the normally incident beam of Eq. (46), substitution into Eq. (7) and then performing the $z, h_{z}$, and $\theta$ integrations gives

$$
\begin{aligned}
B_{l}(h)= & \frac{1}{4 \pi s^{2}\left(1-h^{2}\right)} \exp \left(-h^{2} / 4 s^{2}\right) \int_{-\infty}^{\infty} \mathrm{d} h_{y} \\
& \times \exp \left[-i k x_{0}\left(1-h^{2}-h_{y}{ }^{2}\right)^{1 / 2}\right] \\
& \times \exp \left(-i k h_{y} y_{0}\right) \exp \left(-h_{y}{ }^{2} / 4 s^{2}\right) \\
& \times \exp \left\{-i l \arcsin \left[h_{y}\left(1-h^{2}\right)^{-1 / 2}\right]\right\} .
\end{aligned}
$$

The $h_{y}$ integral in Eq. (49) cannot be evaluated analytically. But for a beam that is not tightly focused (i.e., $s$ $\ll 1$ ) the $\exp \left(-h_{y}{ }^{2} / 4 s^{2}\right)$ factor in the integrand damps rapidly for $h_{y}{ }^{2} \sim 4 s^{2} \ll 1$. For this case we may expand the exponents in the integrand in powers of $h_{y}$ and retain only the linear and quadratic terms. The resulting integral can be evaluated analytically, giving 


$$
\begin{aligned}
B_{l}(h) \approx & \frac{1}{2 s \pi^{1 / 2}\left(1-h^{2}\right)} \\
& \times \frac{\exp \left[-i k x_{0}\left(1-h^{2}\right)^{1 / 2}\right]}{\left[1-2 i s^{2} k x_{0}\left(1-h^{2}\right)^{-1 / 2}\right]^{1 / 2}} \exp \left(-h^{2} / 4 s^{2}\right) \\
& \times \exp \left\{\frac{-s^{2}\left[l\left(1-h^{2}\right)^{-1 / 2}+k y_{0}\right]^{2}}{1-2 i s^{2} k x_{0}\left(1-h^{2}\right)^{-1 / 2}}\right\}
\end{aligned}
$$

Our $h_{y}{ }^{2} \ll 1$ argument is not overly restrictive, since even for $w_{0}=4 \lambda$, which is a moderately tightly localized beam, we have $\exp \left(-h_{y}{ }^{2} / 4 s^{2}\right)=10^{-7}$ when $h_{y}{ }^{2} \approx 0.10$. The derivation of $A_{l}(h)$ for a normally incident $\epsilon$-polarized Gaussian beam proceeds identically.

The beam shape coefficients of relation (50) are intuitively pleasing in a number of ways. First, for $x_{0}=y_{0}$ $=0$, relation (50) reduces to relation (42), which was inferred from the Davis-Barton model. This illustrates that both the Davis-Barton model and the angular spectrum of plane waves model lead to similar beam shape coefficients for a weakly focused beam. But the angular spectrum of plane waves model arrives at the result much more straightforwardly. This is because of the inverse Fourier transform structure of Eqs. (4) and (7). The $\exp \left[-\left(h_{y}{ }^{2}+h_{z}^{2}\right) / 4 s^{2}\right]$ factor in the Fourier spectrum of the beam's amplitude profile in its focal plane evolves into the $\exp \left(-h^{2} / 4 s^{2}\right)$ factor in the beam shape coefficients.

Another feature of relation (50) is somewhat surprising in light of the analogous result for sphere scattering. If the beam is incident off axis on the cylinder such that the peak of the Gaussian profile occurs at $y_{0}$, the beam shape coefficients taken as a function of $l$ reach their peak value when

$$
l=-k y_{0}\left(1-h^{2}\right)^{1 / 2},
$$

whereas for off-axis sphere scattering they reach their peak value when ${ }^{36}$

$$
l+1 / 2=k y_{0}
$$

The two results are quite similar, except that they differ by a minus sign. Equations (51) and (52) are both examples of the localization principle, i.e., the partial wave $l$ is associated with a geometrical light ray traveling parallel to the $x$ axis of Fig. 1 at a distance $\left(y^{2}+z^{2}\right)^{1 / 2} \approx l / k$ away from it. ${ }^{46,47}$ But, for cylinder scattering, positive partial waves are associated with geometrical rays having negative $y_{0}$, and negative partial waves are associated with geometrical rays having positive $y_{0}$.

Based on the success of the angular spectrum of plane waves model in yielding the beam shape coefficients of the normally incident beam of Eq. (46), this method will now be briefly demonstrated for the $\mu$-polarized diagonally incident focused Gaussian beam of Fig. 2. The dominant propagation direction of the beam makes an angle $\xi$ with the $x$ axis as in Eq. (1), and the center of the beam focal waist is at $\left(x_{0}, y_{0}, z_{0}\right)$. The formula for the beam electric field is obtained by rotating Eq. (43) by the angle $-\xi$ in the $x-z$ plane. The $z$ component of the rotated electric field is then

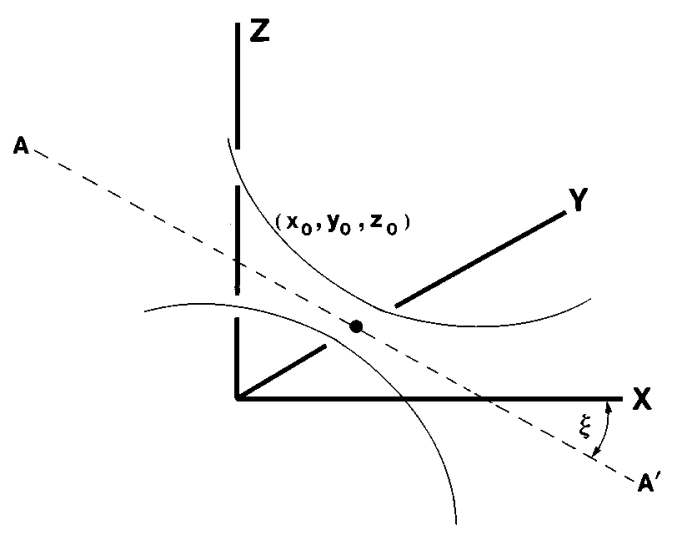

Fig. 2. A focused Gaussian beam has the dominant propagation direction $\mathrm{AA}^{\prime}$ in the $x-z$ plane and making an angle $\xi$ with the $x$ axis. The center of its focal waist is at the coordinate $\left(x_{0}, y_{0}, z_{0}\right)$.

$$
\begin{aligned}
E_{z, \text { inc }}(x, y, z)= & \frac{E_{0} w_{0}^{2}}{4 \pi} \int_{-\infty}^{\infty} k \mathrm{~d} h_{y} \int_{-\infty}^{\infty} k \mathrm{~d} h_{z} \\
& \times \exp \left[i k h_{x}(x \cos \xi-z \sin \xi\right. \\
& \left.\left.-x_{0} \cos \xi+z_{0} \sin \xi\right)\right] \\
& \times \exp \left[i k h_{y}\left(y-y_{0}\right)\right] \\
& \times \exp \left[i k h_{z}(x \sin \xi+z \cos \xi\right. \\
& \left.\left.-x_{0} \sin \xi-z_{0} \cos \xi\right)\right] \exp \left(-h_{y}{ }^{2} / 4 s^{2}\right) \\
& \times \exp \left(-h_{z}{ }^{2} / 4 s^{2}\right)\left(\cos \xi+\frac{h_{z}}{h_{x}} \sin \xi\right)
\end{aligned}
$$

Substituting into Eq. (7) and then performing the $z, h_{z}$, and $\theta$ integrations give

$$
\begin{aligned}
B_{l}(h)= & \frac{\cos \xi}{4 \pi s^{2}\left(1-h^{2}\right)} \exp \left(-i k h z_{0}\right) \exp \left\{-\left[h^{2} \cos ^{2} \xi\right.\right. \\
& \left.\left.+\left(1-h^{2}\right) \sin ^{2} \xi\right] / 4 s^{2}\right\} \int_{-\infty}^{\infty} \mathrm{d} h_{y} \\
& \times \exp \left[-i k x_{0}\left(1-h^{2}-h_{y}{ }^{2}\right)^{1 / 2}\right] \exp \left(-i k h_{y} y_{0}\right) \\
& \times \exp \left\{-\left[2 h\left(1-h^{2}-h_{y}{ }^{2}\right)^{1 / 2}\right.\right. \\
& \left.\left.\times \sin \xi \cos \xi+h_{y}{ }^{2} \cos ^{2} \xi\right] / 4 s^{2}\right\} \\
& \times \exp \left\{-i l \arcsin \left[h_{y}\left(1-h^{2}\right)^{-1 / 2}\right]\right\}
\end{aligned}
$$

Again the exponents in the integrand are expanded in powers of $h_{y}$, and only the linear and quadratic terms are retained. The resulting integral can be evaluated analytically to give

$$
\begin{aligned}
B_{l}(h) \approx & \frac{\cos \xi}{2 s \pi^{1 / 2}\left(1-h^{2}\right) F^{1 / 2}} \\
& \times \exp \left\{-i k\left[h z_{0}+\left(1-h^{2}\right)^{1 / 2} x_{0}\right]\right\} \\
& \times \exp \left\{-\left[h \cos \xi+\left(1-h^{2}\right)^{1 / 2} \sin \xi\right]^{2} / 4 s^{2}\right\} \\
& \times \exp \left\{-s^{2}\left[l\left(1-h^{2}\right)^{-1 / 2}+k y_{0}\right]^{2} / F\right\}
\end{aligned}
$$


where

$$
\begin{aligned}
F= & (\cos \xi)\left[\cos \xi-h\left(1-h^{2}\right)^{-1 / 2} \sin \xi\right] \\
& -2 i s^{2} k x_{0}\left(1-h^{2}\right)^{-1 / 2} .
\end{aligned}
$$

The derivation of $A_{l}(h)$ for a diagonally incident $\epsilon$-polarized Gaussian beam proceeds identically.

\section{Specification of a Set of Beam Shape Coefficients and the Localized Approximation}

We have seen that the beam shape coefficients of Eqs. (50) and (56) and relation (55) are consistent with a localized interpretation of the incident beam, i.e., the association of each partial wave with a ray trajectory. It was also seen that the approximation of the integrands in Eqs. (49) and (54) was quite accurate for all but the most tightly focused beams. As a result, for the remainder of this paper we take the following as our definition of a $\mu$-polarized diagonally incident focused Gaussian beam:

$$
\begin{aligned}
A_{l}(h)= & 0, \\
B_{l}(h)= & \frac{\cos \xi}{2 s \pi^{1 / 2}\left(1-h^{2}\right) F^{1 / 2}} \\
& \times \exp \left\{-i k\left[h z_{0}+\left(1-h^{2}\right)^{1 / 2} x_{0}\right]\right\} \\
& \times \exp \left\{-\left[h \cos \xi+\left(1-h^{2}\right)^{1 / 2} \sin \xi\right]^{2} / 4 s^{2}\right\} \\
& \times \exp \left\{-s^{2}\left[l\left(1-h^{2}\right)^{-1 / 2}+k y_{0}\right]^{2} / F\right\},
\end{aligned}
$$

where $F$ is given by Eq. (56). Similarly, our definition of an $\epsilon$-polarized diagonally incident focused Gaussian beam is

$$
\begin{aligned}
A_{l}(h)= & \frac{\cos \xi}{2 s \pi^{1 / 2}\left(1-h^{2}\right) F^{1 / 2}} \\
& \times \exp \left\{-i k\left[h z_{0}+\left(1-h^{2}\right)^{1 / 2} x_{0}\right]\right\} \\
& \times \exp \left\{-\left[h \cos \xi+\left(1-h^{2}\right)^{1 / 2} \sin \xi\right]^{2} / 4 s^{2}\right\} \\
& \times \exp \left\{-s^{2}\left[l\left(1-h^{2}\right)^{-1 / 2}+k y_{0}\right]^{2} / F\right\} \\
B_{l}(h)= & 0 .
\end{aligned}
$$

As was seen in Subsection 4.B, when considered as a function of $l$, these coefficients reach their peak value at $l=-k y_{0}\left(1-h^{2}\right)^{1 / 2}$, in accordance with the localization principle for cylinder scattering. When considered as a function of $h$, they reach their peak value at

$$
h=-\sin \xi,
$$

which coincides with the propagation direction of the dominant plane wave in the angular spectrum of the beam. If the beam is tightly focused with $w_{0} \gtrsim \lambda$, the beam confinement parameter $s$ is relatively large. As a result, examination of Eqs. (57) and (58) shows that the range of partial waves $\Delta l$ over which the beam shape coefficients are sizable is small, indicating that only a few rays constitute the beam. The range of $h$ parameters, $\Delta h$, over which the beam shape coefficients are sizable is large, indicating that many component plane waves are required for the construction of a tightly localized Gaussian. Conversely, if the beam is loosely focused with $w_{0}$ $\gg \lambda$, then $s$ is relatively small. This results in $\Delta l$ being large, indicating that a large number of rays constitute the beam, and $\Delta h$ being small, indicating that few component plane waves are required for the construction of a loosely localized Gaussian. These properties of the beam shape coefficients lead us to identify the continuous separation index $h$ as the sine of the angle that a component plane wave in the Fourier angular spectrum of the beam makes with the $x$ axis. We will return to this interpretation of $h$ in Section 6 .

\section{FAR-ZONE FIELDS AND INTENSITY}

In this section we substitute the beam shape coefficients of Eqs. (57) and (58) into Eqs. (16)-(19) to obtain the farzone fields and intensity. In analogy to Eqs. (30) and (35) for diagonal-incidence plane-wave-cylinder scattering, we define the angular scattering amplitudes $S_{\epsilon}(\theta, h)$, $S_{\mu}(\theta, h)$, and $S_{q}(\theta, h)$ as

$$
\begin{aligned}
S_{\epsilon}(\theta, h)= & \sum_{l=-\infty}^{\infty}[\exp (i l \theta)] a_{l}(h) \\
& \times \exp \left\{-s^{2}\left[l\left(1-h^{2}\right)^{-1 / 2}+k y_{0}\right]^{2} / F\right\}, \\
S_{\mu}(\theta, h)= & \sum_{l=-\infty}^{\infty}[\exp (i l \theta)] b_{l}(h) \\
& \times \exp \left\{-s^{2}\left[l\left(1-h^{2}\right)^{-1 / 2}+k y_{0}\right]^{2} / F\right\}, \\
S_{q}(\theta, h)= & \sum_{l=-\infty}^{\infty}[\exp (i l \theta)] q_{l}(h) \\
& \times \exp \left\{-s^{2}\left[l\left(1-h^{2}\right)^{-1 / 2}+k y_{0}\right]^{2} / F\right\} .
\end{aligned}
$$

Assuming that the incident beam is $\mu$ polarized, substitution of Eqs. (11), (57), and (60) into the expression for $T_{1}$ in Eqs. (18) gives

$$
\begin{aligned}
T_{1}(r, \theta, z)= & \frac{\cos \xi}{2 s \pi^{1 / 2}} \int_{-\infty}^{\infty} \mathrm{d} h \frac{S_{\mu}(\theta, h)}{\left(1-h^{2}\right)^{3 / 4} F^{1 / 2}} \\
& \times \exp \left[i k r\left(1-h^{2}\right)^{1 / 2}\right] \exp (i k h z) \\
& \times \exp \left\{-i k\left[h z_{0}+\left(1-h^{2}\right)^{1 / 2} x_{0}\right]\right\} \\
& \times \exp \left\{-\left[h \cos \xi+\left(1-h^{2}\right)^{1 / 2}\right.\right. \\
& \left.\times \sin \xi]^{2} / 4 s^{2}\right\} .
\end{aligned}
$$

The phase of the integrand in Eq. (61) is

$$
\begin{gathered}
\Phi(h)=k\left(r-x_{0}\right)\left(1-h^{2}\right)^{1 / 2}+k\left(z-z_{0}\right) h \\
+\operatorname{Phase}\left(S_{\mu}\right)+\operatorname{Phase}\left(F^{-1 / 2}\right) .
\end{gathered}
$$

In the far-zone limit $r \rightarrow \infty$ and $z \rightarrow \infty$, with the ratio $z / r$ being fixed, the most rapidly varying contributions to the phase of the integrand in Eq. (61) are the first two terms of Eq. (62), since the phases of $S_{\mu}$ and $F^{1 / 2}$ are independent of the coordinates at which the scattered field is evaluated. With this being the case, Eq. (61) may be evaluated by the method of stationary phase (sp in subscript and superscript) in the far-zone limit, ${ }^{48}$ giving

$$
T_{1}^{\mathrm{sp}}(r, \theta, z)=G_{\mathrm{sp}} S_{\mu}\left(\theta, h_{\mathrm{sp}}\right),
$$

where 


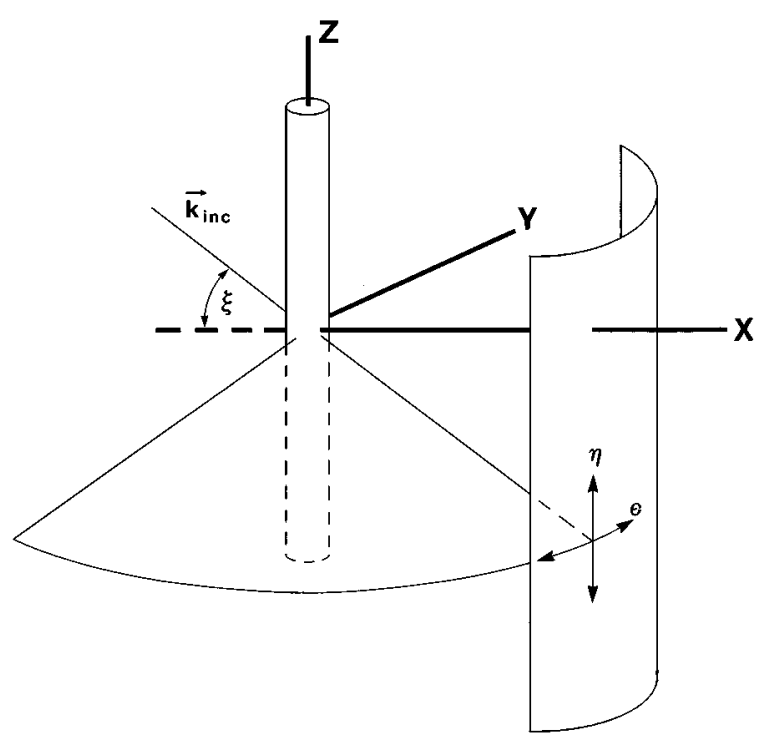

Fig. 3. The horizontal and vertical directions on the viewing screen are $\theta$ and $\eta$, respectively. The scattered wave produced by each plane-wave component in the Fourier spectrum of the incident beam is a cone with a different opening angle and intersecting the viewing screen at a different vertical coordinate $\eta$.

$$
\begin{aligned}
h_{\mathrm{sp}}= & \frac{z}{\left(r^{2}+z^{2}\right)^{1 / 2}}=\sin \eta, \\
G_{\mathrm{sp}}= & \frac{(\cos \xi)(\cos \eta)^{1 / 2} \exp (-i \pi / 4)}{s(2 k r)^{1 / 2}\left[(\cos \xi) \cos (\xi+\eta)-2 i s^{2} k x_{0}\right]^{1 / 2}} \\
& \times \exp \left\{i k\left[\left(r-x_{0}\right)^{2}+\left(z-z_{0}\right)^{2}\right]^{1 / 2}\right\} \\
& \times \exp \left\{-\left[\sin ^{2}(\xi+\eta)\right] / 4 s^{2}\right\} .
\end{aligned}
$$

The angle $\eta$ between the horizontal plane and the line that joins the origin with the field point $(r, \theta, z)$ is shown in Fig. 1. Taken as a function of $\eta$, Eq. (65) reaches its peak value when $\eta=-\xi$, i.e., along the cone which at $\theta=0^{\circ}$ forms the continuation of the dominant propagation direction of the incident beam. This is shown in Fig. 3.

The stationary-phase method can be used to evaluate the other amplitudes of Eqs. (18) for both $\mu$-polarized incidence and $\epsilon$-polarized incidence. For $\mu$-polarized incidence the results are

$$
\begin{aligned}
& T_{2}^{\mathrm{sp}}(r, \theta, z)=G_{\mathrm{sp}} S_{q}\left(\theta, h_{\mathrm{sp}}\right), \\
& T_{3}^{\mathrm{sp}}(r, \theta, z)=G_{\mathrm{sp}}(\cos \eta) S_{\mu}\left(\theta, h_{\mathrm{sp}}\right), \\
& T_{4}^{\mathrm{sp}}(r, \theta, z)=G_{\mathrm{sp}}(\cos \eta) S_{q}\left(\theta, h_{\mathrm{sp}}\right), \\
& T_{5}^{\mathrm{sp}}(r, \theta, z)=G_{\mathrm{sp}}(\sin \eta) S_{\mu}\left(\theta, h_{\mathrm{sp}}\right), \\
& T_{6}^{\mathrm{sp}}(r, \theta, z)=G_{\mathrm{sp}}(\sin \eta) S_{q}\left(\theta, h_{\mathrm{sp}}\right) .
\end{aligned}
$$

The far-zone scattered fields for a $\mu$-polarized incident beam are then

$$
\begin{aligned}
& \lim _{r \rightarrow \infty} \mathbf{E}_{\text {scatt }}(r, \theta, z)=\mathbf{E}_{\mu \mu}(r, \theta, z)+\mathbf{E}_{\mu \epsilon}(r, \theta, z), \\
& \lim \mathbf{B}_{\text {scatt }}(r, \theta, z)=\mathbf{B}_{\mu \mu}(r, \theta, z)+\mathbf{B}_{\mu \epsilon}(r, \theta, z),
\end{aligned}
$$

where the $\mu$-polarized scattered fields are

$$
\begin{aligned}
& \mathbf{E}_{\mu \mu}(r, \theta, z)=\varepsilon_{0}\left[S_{\mu}\left(\theta, h_{\mathrm{sp}}\right)\right]\left[-(\sin \eta) \hat{u}_{r}+(\cos \eta) \hat{u}_{z}\right] \\
& \mathbf{B}_{\mu \mu}(r, \theta, z)=\frac{\varepsilon_{0}}{c}\left[S_{\mu}\left(\theta, h_{\mathrm{sp}}\right)\right]\left(-\hat{u}_{\theta}\right)
\end{aligned}
$$

and the $\epsilon$-polarized scattered fields are

$$
\begin{aligned}
& \mathbf{E}_{\mu \epsilon}(r, \theta, z)=\varepsilon_{0}\left[S_{q}\left(\theta, h_{\mathrm{sp}}\right)\right] \hat{u}_{\theta}, \\
& \mathbf{B}_{\mu \epsilon}(r, \theta, z)=\frac{\varepsilon_{0}}{c}\left[S_{q}\left(\theta, h_{\mathrm{sp}}\right)\right]\left[-(\sin \eta) \hat{u}_{r}+(\cos \eta) \hat{u}_{z}\right],
\end{aligned}
$$

with

$$
\begin{aligned}
\varepsilon_{0}= & \frac{i E_{0} w_{0}(\cos \eta)^{1 / 2}}{\pi^{1 / 2} r\left[\cos \xi \cos (\xi+\eta)-2 i s^{2} k x_{0}\right]^{1 / 2}} \\
& \times \exp \left\{i k\left[\left(r-x_{0}\right)^{2}+\left(z-z_{0}\right)^{2}\right]^{1 / 2}\right\} \\
& \times \exp \left\{-\left[\sin ^{2}(\xi+\eta)\right] / 4 s^{2}\right\} .
\end{aligned}
$$

The far-zone scattered intensity is

$$
\begin{aligned}
\lim _{r \rightarrow \infty} \mathbf{I}_{\text {scatt }}(r, \theta, z)= & \frac{E_{0}{ }^{2} w_{0}{ }^{2} \cos \eta}{\pi r^{2}}\left[\cos ^{2} \xi \cos ^{2}(\xi+\eta)\right. \\
& \left.+4 s^{2} x_{0}{ }^{2} / w_{0}{ }^{2}\right]^{-1 / 2} \\
& \times\left(\left\{\exp -\left[\sin ^{2}(\xi+\eta)\right] / 2 s^{2}\right\}\right) \\
& \times\left[\left|S_{\mu}\left(\theta, h_{\mathrm{sp}}\right)\right|^{2}+\left|S_{q}\left(\theta, h_{\mathrm{sp}}\right)\right|^{2}\right] \\
& \times\left[(\cos \eta) \hat{u}_{r}+(\sin \eta) \hat{u}_{z}\right] .
\end{aligned}
$$

For an $\epsilon$-polarized incident beam the stationary-phase evaluation of $T_{1}$ through $T_{6}$ gives

$$
\begin{aligned}
& T_{1}^{\mathrm{sp}}(r, \theta, z)=-G_{\mathrm{sp}} S_{q}\left(\theta, h_{\mathrm{sp}}\right), \\
& T_{2}^{\mathrm{sp}}(r, \theta, z)=G_{\mathrm{sp}} S_{\epsilon}\left(\theta, h_{\mathrm{sp}}\right), \\
& T_{3}^{\mathrm{sp}}(r, \theta, z)=-G_{\mathrm{sp}}(\cos \eta) S_{q}\left(\theta, h_{\mathrm{sp}}\right), \\
& T_{4}^{\mathrm{sp}}(r, \theta, z)=G_{\mathrm{sp}}(\cos \eta) S_{\epsilon}\left(\theta, h_{\mathrm{sp}}\right), \\
& T_{5}^{\mathrm{sp}}(r, \theta, z)=-G_{\mathrm{sp}}(\sin \eta) S_{q}\left(\theta, h_{\mathrm{sp}}\right), \\
& T_{6}^{\mathrm{sp}}(r, \theta, z)=G_{\mathrm{sp}}(\sin \eta) S_{\epsilon}\left(\theta, h_{\mathrm{sp}}\right) .
\end{aligned}
$$

The far-zone scattered fields for an $\epsilon$-polarized incident beam are then

$$
\begin{aligned}
& \lim _{r \rightarrow \infty} \mathbf{E}_{\text {scatt }}(r, \theta, z)=\mathbf{E}_{\epsilon \epsilon}(r, \theta, z)-\mathbf{E}_{\epsilon \mu}(r, \theta, z), \\
& \lim _{r \rightarrow \infty} \mathbf{B}_{\text {scatt }}(r, \theta, z)=\mathbf{B}_{\epsilon \epsilon}(r, \theta, z)-\mathbf{B}_{\epsilon \mu}(r, \theta, z),
\end{aligned}
$$

where the $\epsilon$-polarized scattered fields are

$$
\begin{aligned}
& \mathbf{E}_{\epsilon \epsilon}(r, \theta, z)=\varepsilon_{0}\left[S_{\epsilon}\left(\theta, h_{\mathrm{sp}}\right)\right] \hat{u}_{\theta}, \\
& \mathbf{B}_{\epsilon \epsilon}(r, \theta, z)=\frac{\varepsilon_{0}}{c}\left[S_{\epsilon}\left(\theta, h_{\mathrm{sp}}\right)\right]\left[-(\sin \eta) \hat{u}_{r}+(\cos \eta) \hat{u}_{z}\right]
\end{aligned}
$$

and the $\mu$-polarized scattered fields are 


$$
\begin{aligned}
& \mathbf{E}_{\epsilon \mu}(r, \theta, z)=\varepsilon_{0}\left[S_{q}\left(\theta, h_{\mathrm{sp}}\right)\right]\left[-(\sin \eta) \hat{u}_{r}+(\cos \eta) \hat{u}_{z}\right], \\
& \mathbf{B}_{\epsilon \mu}(r, \theta, z)=\frac{\varepsilon_{0}}{c}\left[S_{q}\left(\theta, h_{\mathrm{sp}}\right)\right]\left(-\hat{u}_{\theta}\right) .
\end{aligned}
$$

The far-zone scattered intensity is

$$
\begin{aligned}
\lim _{r \rightarrow \infty} \mathbf{I}_{\text {scatt }}(r, \theta, z)= & \frac{E_{0}{ }^{2} w_{0}{ }^{2} \cos \eta}{\pi r^{2}}\left[\cos ^{2} \xi \cos ^{2}(\xi+\eta)\right. \\
& \left.+4 s^{2} x_{0}{ }^{2} / w_{0}{ }^{2}\right]^{-1 / 2} \\
& \times\left(\left\{\exp -\left[\sin ^{2}(\xi+\eta)\right] / 2 s^{2}\right\}\right) \\
& \times\left[\left|S_{\epsilon}\left(\theta, h_{\mathrm{sp}}\right)\right|^{2}+\left|S_{q}\left(\theta, h_{\mathrm{sp}}\right)\right|^{2}\right] \\
& \times\left[(\cos \eta) \hat{u}_{r}+(\sin \eta) \hat{u}_{z}\right] .
\end{aligned}
$$

Equations (67)-(71) and (73)-(76) are the principal results of this paper and should be compared with the diagonally incident plane-wave results of Eqs. (25)-(36). It is interesting to see that even though we employed cylindrical coordinates in the derivation of the Gaussian-beam scattering equations, the results exhibit features characteristic of scattering by a localized source. For a cylindrical source, conservation of energy dictates that the scattered fields fall as $r^{-1 / 2}$ and the scattered intensity falls as $r^{-1}$. This behavior is evident in Eqs. (16), (17), and (19) and Eqs. (28), (31), and (36) for plane-wave incidence. But a localized source, such as the small portion the cylinder illuminated by a focused Gaussian beam, produces scattered fields that fall as $r^{-1}$ and a scattered intensity that falls as $r^{-2}$. This behavior is evident in Eqs. (70), (71), and (76). Similarly, the far-zone scattered intensity vector points in the radially outward direction, which is also characteristic of radiation from a localized source.

From a computational point of view Eqs. (71) and (76) require only minor modifications to existing diagonally incident plane-wave-cylinder scattering computer programs. This is especially true if one is interested in a normally incident Gaussian beam focused at the off-axis position $\left(0, y_{0}, 0\right)$. In this case the normalized scattered intensity for diagonal plane-wave scattering with incidence angle $\eta$ is multiplied by an overall factor of $\exp \left[-\left(\sin ^{2} \eta\right) / 2 s^{2}\right]$, and the terms in the sums from $-\infty$ to $\infty$ of Eqs. (30) and (35) are multiplied by $\exp \left[-s^{2}\left(k y_{0}\right.\right.$ $\left.+l / \cos \eta)^{2}\right]$. This gives each sum both cosine and sine dependence for $y_{0} \neq 0$. The scattered intensity is then computed as a function of $\theta$ and $\eta$ as in Fig. 3 .

Further insight into the scattering process is obtained by comparing the scattered fields of Eqs. (67)-(70) and (73)-(75) with the far-zone diffractive spreading of the incident beam in the near-forward direction for the special case of normal incidence $\left(\xi=0^{\circ}\right)$ and with the center of the beam focal waist taken to be at the on-axis position $\left(x_{0}=y_{0}=z_{0}=0\right)$. For the $\mu$-polarized normally incident beam of Eqs. (37), for example, the far-zone nearforward-direction beam fields become

$$
\begin{aligned}
\lim _{r \rightarrow \infty} \lim _{\theta, \eta \ll 1} \mathbf{E}_{\text {inc }}(r, \theta, z)= & \frac{-i k E_{0} w_{0}{ }^{2}}{2 r}\left\{\exp \left[i k\left(r^{2}+z^{2}\right)^{1 / 2}\right]\right. \\
& \left.\times \exp \left[-\left(\theta^{2}+\eta^{2}\right) / 4 s^{2}\right]\right\} \\
& \times\left(-\eta \hat{u}_{r}+\hat{u}_{z}\right),
\end{aligned}
$$

$$
\begin{aligned}
\lim _{r \rightarrow \infty} \lim _{\theta, \eta \ll 1} \mathbf{B}_{\text {inc }}(r, \theta, z)= & \frac{-i k E_{0} w_{0}^{2}}{2 c r}\left\{\exp \left[i k\left(r^{2}+z^{2}\right)^{1 / 2}\right]\right. \\
& \left.\times \exp \left[-\left(\theta^{2}+\eta^{2}\right) / 4 s^{2}\right]\right\} \\
& \times\left(-\hat{u}_{\theta}\right),
\end{aligned}
$$

whereas Eqs. (68) and (69) for the scattered fields reduce in this limit to

$$
\begin{aligned}
\mathbf{E}_{\mu \mu}(r, \theta, z) \approx & \frac{i E_{0} w_{0}}{\pi^{1 / 2} r}\left\{\exp \left[i k\left(r^{2}+z^{2}\right)^{1 / 2}\right]\right. \\
& \left.\times\left[\exp \left(-\eta^{2} / 4 s^{2}\right)\right]\right\} \\
& \times\left[S_{\mu}(\theta, \eta)\right]\left(-\eta \hat{u}_{r}+\hat{u}_{z}\right), \\
\mathbf{B}_{\mu \mu}(r, \theta, z) \approx & \frac{i E_{0} w_{0}}{\pi^{1 / 2} c r}\left\{\exp \left[i k\left(r^{2}+z^{2}\right)^{1 / 2}\right]\right. \\
& \left.\times\left[\exp \left(-\eta^{2} / 4 s^{2}\right)\right]\right\}\left[S_{\mu}(\theta, \eta)\right]\left(-\hat{u}_{\theta}\right), \\
\mathbf{E}_{\mu \epsilon}(r, \theta, z) \approx & \frac{i E_{0} w_{0}}{\pi^{1 / 2} r}\left\{\exp \left[i k\left(r^{2}+z^{2}\right)^{1 / 2}\right]\right. \\
& \left.\times\left[\exp \left(-\eta^{2} / 4 s^{2}\right)\right]\right\}\left[S_{q}(\theta, \eta)\right] \hat{u}_{\theta}, \\
\mathbf{B}_{\mu \epsilon}(r, \theta, z) \approx & \frac{i E_{0} w_{0}}{\pi^{1 / 2} c r}\left\{\exp \left[i k\left(r^{2}+z^{2}\right)^{1 / 2}\right]\right. \\
& \left.\times\left[\exp \left(-\eta^{2} / 4 s^{2}\right)\right]\right\}\left[S_{q}(\theta, \eta)\right] \\
& \times\left(-\eta \hat{u}_{r}+\hat{u}_{z}\right),
\end{aligned}
$$

with Eqs. (60) reducing to

$$
\begin{aligned}
& S_{\mu}(\theta, \eta)=\sum_{l=-\infty}^{\infty}[\exp (i l \theta)]\left[b_{l}\left(h_{\mathrm{sp}}\right)\right] \exp \left(-s^{2} l^{2} / \cos ^{2} \eta\right), \\
& S_{q}(\theta, \eta)=\sum_{l=-\infty}^{\infty}[\exp (i l \theta)]\left[q_{l}\left(h_{\mathrm{sp}}\right)\right] \exp \left(-s^{2} l^{2} / \cos ^{2} \eta\right) .
\end{aligned}
$$

We interpret these equations in the following way. In Eqs. (77) the $\exp \left[-\left(\theta^{2}+\eta^{2}\right) / 4 s^{2}\right]$ factor is the Fraunhofer-diffracted amplitude of the focal plane incident-beam profile. The scattering process replaces the Fraunhofer-diffracted amplitude in the $\theta$ direction of Fig. 3 with the angular scattering amplitudes $S_{\mu}(\theta, \eta)$ and $S_{q}(\theta, \eta)$. But scattering only slowly modulates the Fraunhofer-diffracted amplitude in the $\eta$ direction of Fig. 3 , as was mentioned in the preceding paragraph. This scattering behavior in the near-forward direction is qualitatively illustrated in Fig. 4 and is analogous to the action of a cylindrical lens on a normally incident beam. The lens strongly deflects the rays incident on it in the plane perpendicular to its axis. But it has no effect on the rays incident on it in the plane that contains the lens axis.

Lastly, the scattered fields of relations (78) and (79) are weaker than the incident-beam fields of Eqs. (77) by a factor of $-2 s / \pi^{1 / 2}$. For a tightly focused beam, where $s$ is relatively large, this leads to near-complete destructive interference of the beam and the scattered wave in the near-forward direction in the far zone. This destructive 


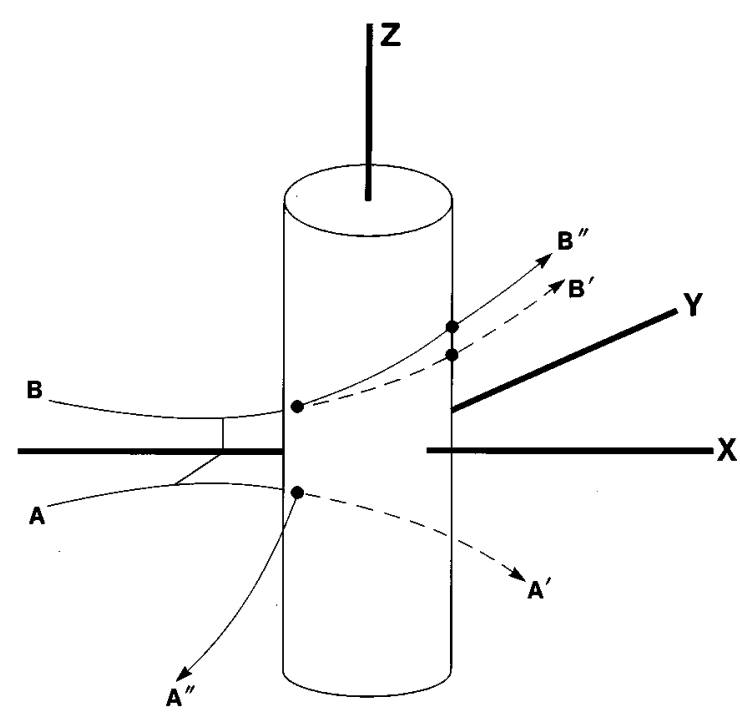

Fig. 4. The beam ray $\mathrm{AA}^{\prime}$ lies in the $x-y$ plane. Upon interaction with the cylinder, its trajectory is strongly deflected to the $\mathrm{A}^{\prime \prime}$ direction. The beam ray $\mathrm{BB}^{\prime}$ lies in the vertical $(x-z)$ plane. Upon transmission through the cylinder, its trajectory is only mildly shifted to $\mathrm{B}^{\prime \prime}$ by multiple internal reflections.

interference produces the diffractive shadow behind the cylinder. ${ }^{49-52}$ The situation is similar for an $\epsilon$-polarized normally incident beam.

\section{DISCUSSION}

In Eqs. (4) and (7) we saw that the partial-wave beam shape coefficients of a monochromatic arbitrary profile incident beam are an inverse Fourier transform of the $z$ component of the beam fields. When we exploit this fact by modeling the incident beam as a sum (i.e., an integral over $h_{y}$ and $h_{z}$ ) of plane waves with different amplitudes (i.e., $\left.\exp \left[-\left(h_{y}{ }^{2}+h_{z}{ }^{2}\right) / 4 s^{2}\right]\right)$ and traveling in different directions (i.e., with the direction cosines $h_{x}, h_{y}$, and $h_{z}$ ), the scattered fields echo the same structure. They become a sum (i.e., an integral over $h$ ) of outgoing conical waves with different amplitudes [i.e., $\exp \left(-\left[\sin ^{2}(\xi\right.\right.$ $+\eta)] / 4 s^{2}$ )] and with different opening angles (i.e., $\sin \eta$ $=h)$. At a given field point $(r, \theta, z)$ in the far zone all the outgoing conical waves destructively interfere except for those centered about $h=r /\left(r^{2}+z^{2}\right)^{1 / 2}$. Thus the scattered intensity at each vertical coordinate $\eta$ on the viewing screen in Fig. 3 contains the imprint of a single plane-wave component in the Fourier angular spectrum of the incident beam. As a function of $\eta$, the scattered intensity was seen in Section 5 to be roughly proportional to the square of the Fourier spectrum of the beam's focal plane amplitude profile. It is centered about $\eta=-\xi$, corresponding to the direction of the dominant planewave component of the beam. For each $\eta$ the Fourier spectrum component is modulated by the cylinder's angular scattering pattern in the $\theta$ direction. This angular scattering pattern is not that of a plane wave [i.e., Eqs. (30) and (35) are a discrete Fourier transform of the plane-wave partial-wave scattering amplitudes $a_{l}, b_{l}$, and $q_{l}$ ]. Rather, the angular scattering pattern of Eqs. (60) is a discrete Fourier transform of the plane-wave partial-wave scattering amplitudes modulated by the Gaussian profile of the beam. Though this interpretation of the scattering pattern as a function of $\eta$ and $\theta$ resulted from my analysis of an incident Gaussian beam, I conjecture that the scattered intensity at each vertical coordinate $\eta$ on the viewing screen for arbitrary beam incidence will again contain the imprint of a single plane-wave component in the Fourier spectrum of the incident beam.

This interpretation of Gaussian-beam-cylinder scattering provides an understanding of a number of different phenomena. For example, a plane wave normally incident on a cylinder produces no cross-polarized scattering. But a focused Gaussian beam normally incident on a cylinder does produce cross-polarized scattering both above and below the horizontal plane. This is because scattering in those directions is due to plane-wave components in the angular spectrum of the Gaussian beam corresponding to diagonal incidence. Similar cross-polarization effects for morphology-dependent resonances are examined in a companion paper. ${ }^{53}$

As a final thought, in Section 4 we examined three different models for the focused Gaussian beam. For a mildly localized beam with $w_{0} \gg \lambda$ the beam shape coefficients for each of the three models are virtually identical. But for a tightly focused beam with $w_{0} \gtrsim \lambda$ there are substantial differences among them. The question then arises as to which, if any, of these models describes an actual laser beam that has been tightly focused by a diffraction-limited lens. In actuality, perhaps none of the three models perfectly describes such a beam. ${ }^{54-56}$ Despite this, I feel justified in examining cylinder scattering in this paper by using the specification-of-coefficients beam model. This is because it gives clean, simple, and readily interpretable results for the far-zone scattered fields and because it suggests a method for generalization to beams of arbitrary profile.

\section{REFERENCES}

1. H. C. van de Hulst, Light Scattering by Small Particles (Dover, New York, 1981), pp. 119-126.

2. M. Kerker, The Scattering of Light and Other Electromagnetic Radiation (Academic, New York, 1969), pp. 39-49.

3. C. F. Bohren and D. R. Huffman, Absorption and Scattering of Light by Small Particles (Wiley, New York, 1983), pp. 83-101.

4. P. Debye, "Das elektromagnetische feld um einen zylinder un die theorie des regenbogens," Phys. Z. 9, 775-778 (1908); reprinted and translated into English in Selected Papers on Geometrical Aspect of Scattering, P. L. Marston, ed., Vol. MS89 of SPIE Milestone Series (Society of PhotoOptical Instrumentation Engineers, Bellingham, Wash., 1994), pp. 198-204.

5. B. van der Pol and H. Bremmer, "The diffraction of electromagnetic waves from an electrical point source round a finitely conducting sphere, with applications to radiotelegraphy and the theory of the rainbow," Philos. Mag. 24, 141176, 825-864 (1937).

6. G. N. Watson, "The diffraction of electric waves by the earth," Proc. R. Soc. London, Ser. A 95, 83-99 (1918); reprinted in Ref. 4, pp. 262-270.

7. H. M. Nussenzveig, "High-frequency scattering by a transparent sphere. I. Direct reflection and transmission," J. Math. Phys. 10, 82-124 (1969).

8. Ref. 1, pp. 210-214. 
9. J. R. Wait, "Scattering of a plane wave from a circular dielectric cylinder at oblique incidence," Can. J. Phys. 33, 189-195 (1955).

10. Ref. 2, pp. $255-266$.

11. Ref. 3, pp. 194-204.

12. N. G. Alexopoulos and P. K. Park, "Scattering of waves with normal amplitude distribution from cylinders," IEEE Trans. Antennas Propag. AP-20, 216-217 (1972).

13. T. Kojima and Y. Yanagiuchi, "Scattering of an offset twodimensional Gaussian beam wave by a cylinder," J. Appl. Phys. 50, 41-46 (1979).

14. S. Kozaki, "Scattering of a Gaussian beam by a homogeneous dielectric cylinder," J. Appl. Phys. 53, 7195-7200 (1982).

15. S. Kozaki, "A new expression for the scattering of a Gaussian beam by a conducting cylinder," IEEE Trans. Antennas Propag. AP-30, 881-887 (1982).

16. T. Kojima and Y. Yanagiuchi, "Scattering of an offset twodimensional Gaussian beam wave by a cylinder," J. Appl. Phys. 50, 41-46 (1979).

17. E. Zimmerman, R. Dandliker, N. Souli, and B. Krattiger, "Scattering of an off-axis Gaussian beam by a dielectric cylinder compared with a rigorous electromagnetic approach," J. Opt. Soc. Am. A 12, 398-403 (1995).

18. S. Kozaki, "Scattering of a Gaussian beam by an inhomogeneous dielectric cylinder," J. Opt. Soc. Am. 72, 1470-1474 (1982).

19. T. C. K. Rao, "Scattering by a radially inhomogeneous cylindrical dielectric shell due to an incident Gaussian beam," Can. J. Phys. 67, 471-475 (1989).

20. A. Z. Elsherbeni, M. Hamid, and G. Tian, "Iterative scattering of a Gaussian beam by an array of circular conducting and dielectric cylinders," J. Elect. Waves Appl. 7, 13231342 (1993)

21. G. Gouesbet and G. Gréhan, "Interaction between shaped beams and an infinite cylinder, including a discussion of Gaussian beams," Part. Part. Syst. Charact. 11, 299-308 (1994).

22. G. Gouesbet and G. Gréhan, "Interaction between a Gaussian beam and an infinite cylinder with the use of non- $\Sigma$ separable potentials," J. Opt. Soc. Am. A 11, 3261-3273 (1994).

23. G. Gouesbet, "The separability theorem revisited with applications to light scattering theory," J. Opt. (Paris) 26, 123-135 (1995).

24. G. Goeusbet, "Interaction between Gaussian beams and infinite cylinders, by using the theory of distributions," J. Opt. (Paris) 26, 225-239 (1995)

25. G. Gouesbet, "Scattering of a first-order Gaussian beam by an infinite cylinder with arbitrary location and arbitrary orientation," Part. Part. Syst. Charact. 12, 242-256 (1995).

26. G. Gouesbet, "Scattering of higher order Gaussian beams by an infinite cylinder," submitted to J. Opt. (Paris).

27. G. Gouesbet, "Interaction between an infinite cylinder and an arbitrary shaped beam," Appl. Opt. (to be published).

28. L. W. Davis, "Theory of electromagnetic beams," Phys. Rev. A 19, 1177-1179 (1979).

29. J. P. Barton and D. R. Alexander, "Fifth-order corrected electromagnetic field components for a fundamental Gaussian beam," J. Appl. Phys. 66, 2800-2802 (1989).

30. G. Gouesbet, G. Gréhan, and B. Maheu, "Scattering of a Gaussian beam by a Mie scatter center using a Bromwich formalism," J. Opt. (Paris) 16, 83-93 (1985).

31. G. Gouesbet, B. Maheu, and G. Gréhan, "Light scattering from a sphere arbitrarily located in a Gaussian beam, using a Bromwich formulation," J. Opt. Soc. Am. A 5, 1427-1443 (1988).

32. J. P. Barton, D. R. Alexander, and S. A. Schaub, "Internal and near-surface electromagnetic fields for a spherical par- ticle irradiated by a focused laser beam,” J. Appl. Phys. 64, 1632-1639 (1988).

33. J. A. Lock, "Contribution of high-order rainbows to the scattering of a Gaussian laser beam by a spherical particle," J. Opt. Soc. Am. A 10, 693-706 (1993).

34. J. A. Lock and G. Gouesbet, "Rigorous justification of the localized approximation to the beam-shape coefficients in generalized Lorenz-Mie theory. I. On-axis beams," J. Opt. Soc. Am. A 11, 2503-2515 (1994).

35. G. Gouesbet and J. A. Lock, "Rigorous justification of the localized approximation to the beam-shape coefficients in generalized Lorenz-Mie theory. II. Off-axis beams," J. Opt. Soc. Am. A 11, 2516-2525 (1994).

36. J. A. Lock, "Improved Gaussian beam-scattering algorithm," Appl. Opt. 34, 559-570 (1995).

37. J. W. Goodman, Introduction to Fourier Optics (McGrawHill, New York, 1968), pp. 48-51.

38. S. Kozaki and H. Sakurai, "Characteristics of a Gaussian beam at a dielectric interface," J. Opt. Soc. Am. 68, 508-514 (1978).

39. E. E. M. Khaled, S. C. Hill, and P. W. Barber, "Scattered and internal intensity of a sphere illuminated with a Gaussian beam," IEEE Trans. Antennas Propag. 41, 295303 (1993).

40. Ref. 1, p. 297, Fig. 64.

41. Ref. 2, p. 256, Fig. 6.1.

42. M. Kerker, D. Cooke, W. A. Farone, and R. A. Jacobsen, "Electromagnetic scattering from an infinite circular cylinder at oblique incidence. I. Radiance functions for $m$ = 1.46," J. Opt. Soc. Am. 56, 487-491 (1966).

43. G. Gréhan B. Maheu, and G. Gouesbet, "Scattering of laser beams by Mie scatter centers: numerical results using a localized approximation,” Appl. Opt. 25, 3539-3548 (1986).

44. G. Gouesbet, G. Gréhan, and B. Maheu, "Localized interpretation to compute all the coefficients $g_{n}^{m}$ in the generalized Lorenz-Mie theory," J. Opt. Soc. Am. A 7, 998-1007 (1990).

45. G. Gouesbet, J. A. Lock, and G. Gréhan, "Partial-wave representations of laser beams for use in light-scattering calculations," Appl. Opt. 34, 2133-2143 (1995).

46. Ref. 1, pp. 208-209.

47. B. Maheu, G. Gréhan, and G. Gouesbet, "Ray localization in Gaussian beams," Opt. Commun. 70, 259-262 (1989).

48. H. Chew, D. D. Cooke, and M. Kerker, "Raman and fluorescent scattering by molecules embedded in dielectric cylinders," Appl. Opt. 19, 44-52 (1980).

49. L. Brillouin, "The scattering cross section of spheres for electromagnetic waves," J. Appl. Phys. 20, 1110-1115 (1949).

50. H. M. Nussenzveig, "High-frequency scattering by an impenetrable sphere," Ann. Phys. (N.Y.) 34, 23-95 (1965); see Fig. 14 on p. 82.

51. J. A. Lock and E. A. Hovenac, "Diffraction of a Gaussian beam by a spherical obstacle," Am. J. Phys. 61, 698-707 (1993); see Fig. 4 on p. 704.

52. J. A. Lock, "Interpretation of extinction in Gaussian-beam scattering," J. Opt. Soc. Am. A 12, 929-938 (1995).

53. J. A. Lock, "Morphology-dependent resonances of an infinitely long circular cylinder illuminated by a diagonally incident plane wave or a focused Gaussian beam," J. Opt. Soc. Am. A 14, 653-661 (1997)

54. J. T. Hodges, G. Gréhan, G. Gouesbet, and C. Presser, "Forward scattering of a Gaussian beam by a nonabsorbing sphere,” Appl. Opt. 34, 2120-2132 (1995).

55. J. A. Lock and J. T. Hodges, "Far field scattering of an axisymmetric laser beam of arbitrary profile by an on-axis spherical particle," Appl. Opt. 35, 4283-4290 (1996).

56. J. A. Lock and J. T. Hodges, "Far-field scattering of a nonGaussian off-axis axisymmetric laser beam by a spherical particle," Appl. Opt. 35, 6605-6616 (1996). 